\title{
Inlay graft of acellular dermal matrix to prevent incisional dehiscence after radiotherapy in prosthetic breast reconstruction
}

\author{
Mi Jung Kim ${ }^{1}$, Sung Jae Ahn ${ }^{1}$, Kenneth L. Fan ${ }^{2}$, Seung Yong Song ${ }^{1}$, Dae Hyun Lew ${ }^{1}$, Dong Won Lee ${ }^{1}$ \\ ${ }^{1}$ Department of Plastic and Reconstructive Surgery and Institute for Human Tissue Restoration, Yonsei University College of Medicine, Seoul, \\ Korea; ${ }^{2}$ Department of Plastic and Reconstructive Surgery, MedStar Georgetown University Hospital, Washington DC, USA
}

Background As the indications for postmastectomy radiotherapy expand, innovative solutions are required to reduce operative complications and reconstructive failure after prosthetic breast reconstruction. In this study, we investigated the effectiveness of acellular dermal matrix (ADM) inlay grafts in preventing postoperative wound dehiscence of irradiated breasts in the context of prosthetic breast reconstruction.

Methods A retrospective analysis was conducted of 45 patients who received two-stage prosthetic reconstruction and radiotherapy following mastectomy. An ADM graft was placed beneath the incisional site during the second-stage operation in 19 patients using marionette sutures, whereas the control group did not receive the ADM reinforcement. Patient demographics and complications such as wound dehiscence, capsular contracture, peri-prosthetic infection, cellulitis, and seroma were compared between the two groups.

Results During an average follow-up period of 37.1 months, wound dehiscence occurred significantly less often in the ADM-reinforced closure group (0\%) than in the non-ADM group (23.1\%) $(P=0.032)$. There was no significant difference between the two groups in relation to other complications, such as capsular contracture, postoperative infection, or seroma.

Conclusions The ADM inlay graft is a simple and easily reproducible technique for preventing incisional dehiscence in the setting of radiotherapy after prosthetic breast reconstruction. The ADM graft serves as a buttress to offload tension during healing and provides a mechanical barrier against pathogens. Application of this technique may serve to reduce complications in prosthetic breast reconstruction after radiotherapy.

Keywords Mammaplasty / Breast implantation / Acellular dermis / Radiotherapy / Complications
Correspondence: Dong Won Lee Department of Plastic and Reconstructive Surgery, Yonsei University College of Medicine, 50-1 Yonsei-ro, Seodaemun-gu, Seoul 03722, Korea

Tel: $+82-2-2228-2215$

Fax: +82-2-393-6947

E-mail:xyphoss@yuhs.ac

Received: January 24, 2018 - Revised: July 13, $2019 \bullet$ Accepted: September 1, 2019

pISSN: 2234-6163 • elSSN: 2234-6171 • https://doi.org/10.5999/aps.2018.00073• Arch Plast Surg 2019;46:544-549

\section{INTRODUCTION}

Postmastectomy radiation therapy after total mastectomy has been shown to reduce the loco-regional recurrence rate and to increase overall survival in both early and advanced breast cancer patients $[1,2]$. While immediate breast reconstruction may improve quality of life for mastectomy patients, increased complication rates are seen when the reconstructed breast is subject- 
ed to radiotherapy $[3,4]$. In a prospective cohort of irradiated breast cancer patients, the implant-based reconstruction group had double the odds of any complication compared to the autologous breast reconstruction group at 2 years after surgery [3]. The observed complications include capsular contracture, infection, pain, skin necrosis, fibrosis, and impaired wound healing $[5,6]$. In particular, earlier studies reported that wound dehiscence occurred in $10 \%-23.5 \%$ of irradiated breasts, compared to $1 \%-2 \%$ in non-irradiated patients [6-8]. A higher rate of infection is intimately linked to wound dehiscence, as implants are susceptible to infection because seeding can occur even through a small opening. Despite this, implant-based reconstruction appears to be on the rise globally, which is attributable to several factors, such as patients' and physicians' preferences, changes in oncologic practice, and the increased frequency of bilateral mastectomies $[9,10]$. Therefore, developing strategies to mitigate these complications is of paramount importance.

Acellular dermal matrix (ADM) is widely used in implantbased breast reconstruction surgery to cover the inferolateral pole of an implant for superior aesthetic outcomes, as well as in efforts to prevent capsular contracture $[11,12]$. A systematic review found that $\mathrm{ADM}$ usage does not predispose irradiated patients to higher rates of complications when used to aid prosthetic breast reconstruction [13]. However, scant research has explored whether an ADM inlay graft can specifically support the incision site and prevent complications in irradiated breasts. In this study, the primary aim was to determine whether the use of an ADM inlay graft could prevent incisional dehiscence in irradiated breasts. The secondary aim was to determine whether the use of an $\mathrm{ADM}$ inlay graft was related to other complications, such as infection or seroma.

\section{METHODS}

\section{Patient selection}

A retrospective study was conducted of patients who underwent mastectomy followed by two-stage prosthetic breast reconstruction and postmastectomy radiotherapy between July 2012 and August 2016 at our institute. Two cohorts were examined: patients with $(\mathrm{n}=19)$ and without $(\mathrm{n}=26)$ an ADM inlay graft at the incisional site during the second-stage operation. After April 2015, ADM inlay grafts were applied when the amount of closing tension was high after implants were placed, with the intention of reinforcing the wound. Specifically, the incision was deemed to have high tension when it was located directly on or near the point of maximal projection after the permanent implant was inserted. Two plastic surgeons (DWL and SYS) performed all reconstructions. Patient information was collected from hospital electronic medical records, including demographic variables such as age, cancer stage, and body mass index, as well as information regarding the expanded volume of the tissue expander, permanent implant volume, chemotherapy, anti-estrogen therapy, target therapy, and complications. The institutional review board approved this study design and the requirement for informed consent was waived (IRB No. 4-2018-1183).

\section{Reconstruction technique}

All patients underwent total mastectomy or skin-/nipple-sparing mastectomy performed by a breast oncologic surgeon, followed by immediate breast reconstruction by a plastic surgeon. After mastectomy, a tissue expander was inserted into the subpectoral plane and the lower lateral portion of the implant was covered with an $\mathrm{ADM}$ sling. The expander was prefilled with approximately one-third of the mastectomy volume. Expansion began 2 weeks after the operation and proceeded every 2 or 3 weeks. Prior to radiation therapy, the expander was deflated to include only $150-250 \mathrm{~mL}$ of saline to make it less prominent in the supine position, thereby minimizing interference with postmastectomy radiotherapy [14]. Immediately following completion of radiation therapy, re-expansion was performed every 2 or 3 weeks until the desired breast volume was reached.

The second stage of reconstruction took place at least 3 months after the completion of radiation therapy and consisted of tissue expander removal with or without capsulectomy, followed by insertion of a permanent shaped cohesive-gel silicone implant. The incision was made as short as possible $(6 \mathrm{~cm}$ on average), considering the impaired wound healing in irradiated tissues. ADM (MegaDerm; $4 \times 7 \mathrm{~cm}$ and $0.8-1.4 \mathrm{~mm}$ in thickness; L\&C Bio Corp., Seoul, Korea) was laid between the permanent implant and the expanded skin flap at the incisional site as an inlay graft to prevent incisional dehiscence in the $\mathrm{ADM}$ group. The ADM was fixed to the skin flap with Vicryl 3-0 pullout sutures. These marionette sutures were removed on postoperative day 7 (Fig. 1).

\section{Radiation protocol}

Radiation therapy commenced within 6 weeks after mastectomy or completion of the last cycle of chemotherapy. Prior to radiotherapy, all patients underwent simulation computed tomography scans for radiotherapy planning. The clinical target volume, including the ipsilateral chest wall, mastectomy scar, axillary nodes, internal mammary lymph nodes, and supraclavicular lymph nodes, was contoured. Depending on the protocol chosen by the radiation oncology team, patients received either conventional fractionation radiotherapy or hypofractionated radiotherapy. Patients with the former radiotherapy protocol were 


\section{Fig. 1. Acellular dermal matrix inlay graft}

Acellular dermal matrix is laid between the permanent implant and the expanded skin flap on the incisional site in an inlay graft manner. The skin flap, pectoral muscle and previously grafted acellular dermal matrix are sectioned along the meridian line.

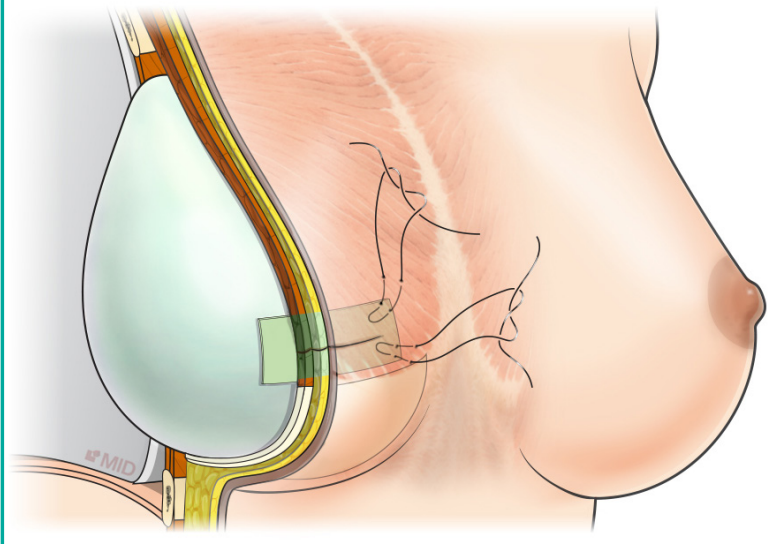

irradiated with $50.4 \mathrm{~Gy}$ in 28 fractions, while the prescription dose of the latter was 40.05 Gy in 15 daily fractions of $2.67 \mathrm{~Gy}$.

\section{Statistical analysis}

When the data followed a normal distribution, continuous data were analyzed with the independent-samples t-test. Otherwise, the nonparametric Mann-Whitney test was used. The chisquare or Fisher exact test was performed to analyze categorical data. Significance was set at $\mathrm{P}<0.05$, using IBM SPSS Statistics for Windows, version 23.0 (IBM Corp., Armonk, NY, USA) for all computations.

\section{RESULTS}

Forty-five breast cancer patients who underwent two-stage prosthetic (expander and implant) reconstruction with postmastectomy radiotherapy were retrospectively examined. The demographic characteristics of patients in the non-ADM inlay graft and the ADM inlay graft group are illustrated in Table 1 . The two groups were similar in age, body mass index, and preoperative breast volume $(\mathrm{P}>0.05)$. There were also no significant differences between groups in the expanded expander volume, permanent implant volume, or the difference between those two values $(P>0.05)$. The two groups showed a similar distribution of additional therapy, such as neoadjuvant chemotherapy, adjuvant chemotherapy, anti-estrogen therapy, and target therapy $(P>0.05)$. However, there was a significant difference between the two groups in the radiation protocol $(\mathrm{P}=0.005)$.

The rates of complications were compared between the two groups (Table 2). Wound dehiscence occurred in six cases in the
Table 1. Patients' demographic characteristics

\begin{tabular}{|lccr|}
\hline Characteristics & $\begin{array}{c}\text { Non-ADM } \\
\text { group } \\
(\mathbf{n}=\mathbf{2 6})\end{array}$ & $\begin{array}{c}\text { ADM } \\
\text { group } \\
(\mathbf{n}=19)\end{array}$ & P-value \\
\hline Age $(\mathrm{yr})$ & $41.4 \pm 10.6$ & $41.1 \pm 10.8$ & 0.894 \\
BMI $\left(\mathrm{kg} / \mathrm{m}^{2}\right)$ & $21.6 \pm 2.9$ & $20.9 \pm 2.5$ & 0.179 \\
Smoking & $2(7.7)$ & $1(5.3)$ & $>0.999$ \\
Interval between second operation & $291.8 \pm 312.2$ & $216.8 \pm 54.5$ & $\left.0.510^{\mathrm{a}}\right)$ \\
$\quad$ and radiation therapy (mon) & & & \\
Preoperative breast volume (mL) & $365.0 \pm 143.5$ & $361.1 \pm 105.7$ & 0.921 \\
Excised mastectomy skin (cm²) & $44.4 \pm 33.2$ & $52.9 \pm 45.1$ & 0.510 \\
Expanded volume (mL) & $443.2 \pm 106.5$ & $439.5 \pm 101.7$ & 0.910 \\
Permanent implant volume (mL) & $358.2 \pm 89.0$ & $313.2 \pm 66.6$ & $0.143^{\text {a) }}$ \\
Final stage & & & 0.052 \\
I & $8(30.8)$ & $3(15.8)$ & \\
II & $11(42.3)$ & $9(47.4)$ & \\
III & $7(26.9)$ & $7(36.8)$ & \\
IV & 0 & 0 & \\
Neoadjuvant chemotherapy & $10(38.5)$ & $10(52.6)$ & 0.379 \\
Adjuvant chemotherapy & $14(53.8)$ & $10(52.6)$ & $>0.999$ \\
Anti-estrogen therapy & $20(76.9)$ & $13(68.4)$ & 0.734 \\
Target therapy & 0 & $2(10.5)$ & 0.173 \\
Radiation protocol & & & 0.005 \\
$\quad$ Conventional radiotherapy & $11(42.3)$ & $16(84.2)$ & \\
Hypofractionated radiotherapy & $15(57.7)$ & $3(15.8)$ & \\
Follow-up period (mon) & $41.7 \pm 16.7$ & $30.7 \pm 4.5$ & 0.171 \\
\hline
\end{tabular}

Values are presented as mean \pm SD or number (\%).

ADM, acellular dermal matrix; BMl, body mass index.

aThe statistical analysis was performed using the Mann-Whitney U test. The rest of the continuous variables were analyzed with the independent-samples t-test.

Table 2. Postoperative complications

\begin{tabular}{|lclc|}
\hline Complications & $\begin{array}{c}\text { Non-ADM } \\
\text { group }(\mathbf{n = 2 6 )}\end{array}$ & $\begin{array}{c}\text { ADM group } \\
(\mathbf{n = 1 9 )}\end{array}$ & P-value \\
\hline Wound dehiscence & $6(23.1)$ & 0 & 0.032 \\
Capsular contracture & $9(34.6)$ & $4(21.1)$ & 0.507 \\
Cellulitis & $1(3.8)$ & $3(15.8)$ & 0.295 \\
Peri-prosthetic infection & $2(7.7)$ & 0 & 0.501 \\
$\begin{array}{l}\text { Seroma/hematoma } \\
\text { Total complications }\end{array}$ & 0 & 0 & - \\
$\begin{array}{l}\text { Needing major revisional } \\
\text { surgery }\end{array}$ & $5(69.2)$ & $7(36.8)$ & 0.031 \\
\hline Values are presented as number (\%). & 0 & 0.063 \\
\hline
\end{tabular}

non-ADM inlay graft group, while no wound dehiscence was observed in the ADM inlay graft group $(\mathrm{P}=0.032)$. Among the six operative revisions that were necessary in the non-ADM inlay graft group, two patients had implants downsized to reduce skin tension. Three other patients underwent reconstruction with a latissimus dorsi flap due to an unhealthy skin condition after repeated repairs. The last patient was treated with debridement, and an ADM inlay graft was used to repair the dehiscence site for therapeutic purposes. Nine cases of capsular contracture (34.6\%) occurred in the non-ADM inlay graft group, and four 
cases of capsular contracture (21.1\%) occurred in the ADM inlay graft group [15]. In the non-ADM group, three patients (9.4\%) suffered from postoperative infections, two of whom had a peri-prosthetic infection instigated by wound dehiscence, which required implant change and a latissimus dorsi flap. In the ADM graft group, three cases (15.8\%) of cellulitis occurred, which were accompanied by redness and heating of the skin envelope; these resolved with intravenous antibiotic therapy within a week. No cases of seroma or hematoma were observed in either group. The total complication rate, excluding wound dehiscence, was $46.2 \%$ in the non-ADM graft group, while it was only $36.8 \%$ in the $\mathrm{ADM}$ graft group $(\mathrm{P}=0.532)$. Furthermore, major revisional surgery to resolve the complications was needed in five patients (19.2\%) in the non-ADM graft group and in none of the patients (0\%) in the ADM graft group, but this difference did not achieve statistical significance $(P=0.063)$.

\section{DISCUSSION}

Previously, breast reconstruction with a prosthetic device combined with irradiation was a relative contraindication due to the high reconstruction failure rate of $37 \%$ and the corresponding negative impact on patient satisfaction $[3,5,16]$. Radiation not only increases complications, such as early wound healing problems and late capsular contracture, but also often results in a high-riding nipple and implant malposition [17]. Recently, complications and aesthetic results in implant-based reconstruction of irradiated breasts have improved to acceptable rates when compared to prior studies, with technical improvements including muscular coverage, use of $\mathrm{ADM}$, newer prostheses, and modern radiation therapy protocols [18]. The use of ADM can provide aesthetic and psychological benefits, without affecting the complication rate in irradiated breasts [13]. Although Pestana et al. [19] suggested that ADM may increase complications requiring reoperation, they did not take into consideration whether radiation therapy took place before or after the reconstruction.

Although the volume of the permanent implants used in this study typically did not exceed that of the expander, tension upon wound closure can also be affected by the relative location of the incision in relation to the point of maximal projection. Wound dehiscence usually occurred at the maximal projection point of the implant, where the skin tension is the highest. Previously, Nahabedian [20] suggested making a new incision at the inframammary fold, instead of using the previous mastectomy incision to decrease the chance of wound dehiscence. Although the inferolateral inframammary fold incision is not remarkable, patients and reconstructive surgeons want to avoid additional scars if possible. In this study, application of ADM to the incisional site as an inlay graft decreased the rate of wound dehiscence. The grafted site of ADM was underneath the expanded skin flap, submuscular capsule, or previously taken $\mathrm{ADM}$ capsule, depending on the position of the incision. The $\mathrm{ADM}$ inlay graft is expected to take well regardless of the graft site, because ADM is grafted onto the capsule, which is a wellvascularized tissue.

Chronic injury of the soft tissue after radiation therapy is considered to occur due to the pathogenic fibroatrophic effect of radiation, which results in low tensile strength of the tissue [21]. Endothelial cell injury, vascular dysfunction, altered expression of bioactive cytokines including transforming growth factor-beta 1 , and oxidative stress lead to excessive extracellular matrix deposition of collagen I and III, a persistent excess of myofibroblasts, and gradual fibroblast rarefaction combined with incomplete cell replacement [22]. As described by Cordeiro et al. [23], two-stage postmastectomy reconstruction with expanders and implants has a higher rate of failure at the second stage, but yields improved aesthetic outcome in the setting of postmastectomy radiation therapy compared with direct-to-implant reconstruction. The ADM inlay graft may be considered a strategy to mitigate these complications and reconstructive failure, as demonstrated by our results. We propose several speculative hypotheses regarding how an $\mathrm{ADM}$ inlay graft may prevent incisional dehiscence. First, the ADM inlay graft may act as a mechanical buttress to offload skin tension in a pants-over-vest fashion. The marionette stitches and ADM graft may relieve tension on the incision, improving blood supply to the wound. In a similar manner, $\mathrm{ADM}$ is often used for repair of ventral hernias to reinforce and offload tension on fascial closures [24]. Secondly, the $\mathrm{ADM}$ inlay graft may serve as a scaffold for wound regeneration, as $\mathrm{ADM}$ is already known to promote chronic wound healing [25]. Third, the ADM inlay graft can act as an additional barrier between the prosthetic and external environment. In non-grafted patients, wound complications may lead to severe peri-prosthetic infections that require operative interventions. The additional barrier provided by the ADM inlay graft reduced operative complications to $0 \%$.

This is the first study, as far as the authors are aware, in which $\mathrm{ADM}$ was placed as an inlay graft manner to prevent wound dehiscence in irradiated breasts. The limitations of this study include its retrospective design, in which the patients were not randomly assigned to groups. Furthermore, as we analyzed a series of consecutive patients, the distribution of the radiation protocol was different between the $\mathrm{ADM}$ and non-ADM groups. Our radiation oncologists began using a hypofractionated radiation technique in the middle of the study period, resulting in an im- 
balance between the two groups in the radiation protocol. However, the relationship between hypofractionated radiotherapy and breast reconstruction has not been delineated [25]. Even if hypofractionated radiotherapy affected the complication rate of reconstructions, we believe that the $\mathrm{ADM}$ inlay graft is an ideal and easy to replicate technique for preventing incisional dehiscence in prosthetic reconstruction in the setting of postmastectomy radiation. Additional prospective trials with larger groups are required in the future for further verification of our findings.

\section{NOTES}

\section{Conflict of interest}

No potential conflict of interest relevant to this article was reported.

\section{Ethical approval}

The study was approved by the Institutional Review Board of Severance Hospital (IRB No. 4-2018-1183) and performed in accordance with the principles of the Declaration of Helsinki.

\section{Author contribution}

Conceptualization: Lee DW. Data curation: Ahn SJ. Formal analysis: Song SY. Funding acquisition: Lee DW. Methodology: Fan KL. Project administration: Lew DH. Visualization: Kim MJ. Writing - original draft: Ahn SJ. Writing - review \& editing: Kim MJ. Approval of final manuscript: all authors.

\section{ORCID}

Mi Jung Kim https://orcid.org/0000-0002-7126-6661

Sung Jae Ahn https://orcid.org/0000-0002-0324-0451

Kenneth L. Fan https://orcid.org/0000-0001-5951-5576

Seung Yong Song https://orcid.org/0000-0002-3145-7463

Dae Hyun Lew https://orcid.org/0000-0002-2625-5664

Dong Won Lee https://orcid.org/0000-0003-0046-3139

\section{REFERENCES}

1. Goldhirsch A, Wood WC, Coates AS, et al. Strategies for subtypes-dealing with the diversity of breast cancer: highlights of the St. Gallen International Expert Consensus on the Primary Therapy of Early Breast Cancer 2011. Ann Oncol 2011;22:1736-47.

2. Budach W, Bolke E, Kammers K, et al. Adjuvant radiation therapy of regional lymph nodes in breast cancer: a metaanalysis of randomized trials-an update. Radiat Oncol 2015; $10: 258$.

3. Jagsi R, Momoh AO, Qi J, et al. Impact of radiotherapy on complications and patient-reported outcomes after breast reconstruction. J Natl Cancer Inst 2018;110:157-65.

4. Elder EE, Brandberg Y, Bjorklund T, et al. Quality of life and patient satisfaction in breast cancer patients after immediate breast reconstruction: a prospective study. Breast 2005; 14:2018.

5. Krueger EA, Wilkins EG, Strawderman M, et al. Complications and patient satisfaction following expander/implant breast reconstruction with and without radiotherapy. Int J Radiat Oncol Biol Phys 2001;49:713-21.

6. Lin KY, Blechman AB, Brenin DR. Implant-based, twostage breast reconstruction in the setting of radiation injury: an outcome study. Plast Reconstr Surg 2012;129:817-23.

7. Nahabedian MY. AlloDerm performance in the setting of prosthetic breast surgery, infection, and irradiation. Plast Reconstr Surg 2009; 124:1743-53.

8. Nava MB, Pennati AE, Lozza L, et al. Outcome of different timings of radiotherapy in implant-based breast reconstructions. Plast Reconstr Surg 2011;128:353-9.

9. Albornoz CR, Bach PB, Mehrara BJ, et al. A paradigm shift in U.S. breast reconstruction: increasing implant rates. Plast Reconstr Surg 2013;131:15-23.

10. Park EH, Min SY, Kim Z, et al. Basic facts of breast cancer in Korea in 2014: the 10-year overall survival progress. J Breast Cancer 2017;20:1-11.

11. Chun YS, Verma K, Rosen H, et al. Implant-based breast reconstruction using acellular dermal matrix and the risk of postoperative complications. Plast Reconstr Surg 2010;125: 429-36.

12. JoAnna Nguyen T, Carey JN, Wong AK. Use of human acellular dermal matrix in implant- based breast reconstruction: evaluating the evidence. J Plast Reconstr Aesthet Surg 2011; 64:1553-61.

13. Clemens MW, Kronowitz SJ. Acellular dermal matrix in irradiated tissue expander/implant-based breast reconstruction: evidence-based review. Plast Reconstr Surg 2012;130 (5 Suppl 2):27S-34S.

14. Kronowitz SJ, Robb GL. Breast reconstruction with postmastectomy radiation therapy: current issues. Plast Reconstr Surg 2004;114:950-60.

15. Spear SL, Baker JL Jr. Classification of capsular contracture after prosthetic breast reconstruction. Plast Reconstr Surg 1995;96:1119-23.

16. McCarthy CM, Klassen AF, Cano SJ, et al. Patient satisfaction with postmastectomy breast reconstruction: a comparison of saline and silicone implants. Cancer 2010;116:558491.

17. Spear SL, Shuck J, Hannan L, et al. Evaluating long-term 
outcomes following nipple-sparing mastectomy and reconstruction in the irradiated breast. Plast Reconstr Surg 2014; 133:605e-614e.

18. Sbitany H, Wang F, Peled AW, et al. Immediate implantbased breast reconstruction following total skin-sparing mastectomy: defining the risk of preoperative and postoperative radiation therapy for surgical outcomes. Plast Reconstr Surg 2014;134:396-404.

19. Pestana IA, Campbell DC, Bharti G, et al. Factors affecting complications in radiated breast reconstruction. Ann Plast Surg 2013;70:542-5.

20. Nahabedian MY. Minimizing incisional dehiscence following 2-stage prosthetic breast reconstruction in the setting of radiation therapy. Gland Surg 2013;2:133-6.

21. Delanian S, Lefaix JL. The radiation-induced fibroatrophic process: therapeutic perspective via the antioxidant path- way. Radiother Oncol 2004;73:119-31.

22. Mikkelsen RB, Wardman P. Biological chemistry of reactive oxygen and nitrogen and radiation-induced signal transduction mechanisms. Oncogene 2003;22:5734-54.

23. Cordeiro PG, Albornoz CR, McCormick B, et al. What is the optimum timing of postmastectomy radiotherapy in two-stage prosthetic reconstruction: radiation to the tissue expander or permanent implant? Plast Reconstr Surg 2015; 135:1509-17.

24. Branch JP. A tendon graft weave using an acellular dermal matrix for repair of the Achilles tendon and other foot and ankle tendons. J Foot Ankle Surg 2011;50:257-65.

25 . Brigido SA. The use of an acellular dermal regenerative tissue matrix in the treatment of lower extremity wounds: a prospective 16-week pilot study. Int Wound J 2006;3:181-7. 